\title{
The role of electrodiagnostic testing in carpal tunnel syndrome
}

\section{Lawrence H. Phillips II, M.D., and Vern C. Juel, M.D.}

Department of Neurology, University of Virginia Health Sciences Center, School of Medicine, Charlottesville, Virginia

Electrodiagnostic testing in patients who have upper-extremity symptoms, which may include carpal tunnel syndrome (CTS), has been the gold standard for diagnosis for many years. Depite their value, these tests are underutilized. The authors examined the use patterns of electrodiagnostic testing at the University of Virginia by reviewing the records of the Electromyography Laboratory for the calendar year 1994. Studies in patients with CTS comprised $15 \%$ of the 1626 studies performed during that time. The mononeuropathy was mild in the majority of cases and most of the patients were referred for testing by specialists. There was a clear referral bias on the part of the primary care physicians, and the severity of mononeuropathy in the patients they referred for testing was significantly greater than in patients referred by specialists.

The data indicate that electrodiagnostic testing has clear value in the evaluation of patients who have upper-extremity symptoms. Despite this fact, primary care physicians appear to underutilize electrodiagnostic testing.

Key Words * carpal tunnel syndrome * electromyography * nerve conduction studies * electrodiagnostic testing

Carpal tunnel syndrome (CTS), or compressive median neuropathy at the transverse carpal ligament, is the mononeuropathy most commonly encountered in clinical practice. It has an estimated prevalence of 125 cases per 100,000 population,[13] although other surveys indicate that the prevalence may be as high as $1 \%$ of the population.[14] The clinical signs and symptoms include hand paresthesias, upper-extremity pain, Tinel's sign on percussion over the median nerve at the wrist, Phalen's sign, median distribution sensory loss, and thenar muscle weakness and atrophy. Many of these clinical features are not unique to CTS and distinguishing among the several causes of upper-extremity signs and symptoms often has important therapeutic implications.

Electrodiagnostic testing, including motor and sensory nerve conduction studies and needle electromyographic (EMG) studies, plays an important role in the evaluation of patients with possible CTS. Electrodiagnostic testing has been shown to have a high degree of sensitivity and specificity in evaluation of CTS.[7] In addition, it may help to identify superimposed or alternative neuropathic conditions mimicking CTS, such as cervical radiculopathy, brachial plexopathy, proximal median neuropathy or other upper limb mononeuropathies, or peripheral neuropathy. When electrodiagnostic testing is normal, this may suggest that nonneuropathic conditions, such as arthritis or tendinitis, are 
responsible for the patient's symptoms.

Despite the clear value of nerve conduction and EMG studies in the evaluation of patients with possible CTS, the tests may be underutilized in clinical practice. Stevens,[12] in a population-based study over a 20 -year period, found that approximately $50 \%$ of patients in Rochester, Minnesota, who were suspected of having CTS on clinical grounds were actually referred for electrodiagnostic testing. In other populations, even fewer patients who are diagnosed with CTS and treated with various modalities, including surgery, are referred for electrodiagnostic testing.[10,11]

Since CTS has become an important public health problem because of its frequent occurrence in the setting of repetitive stress injury in the workplace, its diagnosis and treatment have attracted the attention of organized medicine and governmental agencies. At least nine medical specialty societies have published position papers of various sorts, and there have been statements issued by three governmental agencies on the subject.[4] There is increasing debate about the proper setting for electrodiagnostic testing, and some attempt has been made to limit its use in the diagnosis of CTS. Much of this debate has been based on the opinions of individuals or groups with varying degrees of expertise in the field; little has been based on actual data. We performed this study to determine what the actual real-life experience of a clinical neurophysiology laboratory is in the evaluation of CTS.

\section{CLINICAL MATERIAL AND METHODS}

The Electromyography Laboratory staff in the Department of Neurology at the University of Virginia have maintained a custom-programed computer database derived from all studies conducted since 1991. The data routinely input to this database include demographic information, referral information, electrodiagnostic study data, and final diagnosis for each patient examined. A data retrieval routine that allows easy retrieval of any data field in the database is a part of the program. For this study, we retrieved the data obtained in each patient who was either referred to the laboratory for a possible diagnosis of CTS or in whom a final diagnosis of CTS was made as a result of the examination. The study period was January 1, 1994 through December 31, 1994.

For any patient considered to have CTS, studies are routinely performed according to the guidelines jointly published by the American Association of Electrodiagnostic Medicine, the American Academy of Neurology, and the American Academy of Physical Medicine and Rehabilitation as a practice parameter for the diagnosis of CTS.[1-3] These studies routinely include sensory and motor nerve conduction in median and ulnar nerves and a survey needle EMG examination of upper-extremity muscles sufficient to exclude or discover other neuropathic causes for upper-extremity symptoms. Data obtained in all studies for each patient were tabulated in a spreadsheet for analysis. Statistical comparison between groups was performed using the Mann-Whitney ranked sum test.

\section{RESULTS}

During the calendar year 1994, 1626 patients were studied in the Electromyography Laboratory. Of these 1626 patients, $192(11.8 \%)$ were referred for a diagnosis of possible CTS. Of this group, $128(66.7 \%)$ were found to have CTS by the most sensitive electrodiagnostic criteria. The diagnoses in the remaining 64 patients are shown in Table 1. 


\begin{tabular}{|cc|}
\hline TABLE 1 \\
DIAGNOSIS IN PATIENTS FOUND NOT TO HAYE CTS \\
\hline \hline Diagnosis & No. of Patierts \\
\hline normal study & 51 \\
cervical radiculopathy & 5 \\
other (trachial plewopathy, other & 8 \\
mononeuropathy, peripheral neuro pathy & \\
\hline
\end{tabular}

In addition to patients referred for a possible diagnosis of CTS, 135 patients who had been referred for other possible diagnoses were also found to have CTS. Their referral diagnoses are listed in Table 2. A total of 250 patients were found by means of electrodiagnostic criteria to have CTS, but only 128 patients $(51.2 \%)$ were actually thought to have CTS by their referring physician.

\begin{tabular}{|cc|}
\hline \multicolumn{2}{|c|}{ TABLE 2 } \\
REFERRAL DIAGNOS IN IN PATIENTS FOUND TO HAYE CTS \\
\hline \hline Refiral Diagnosis & No. of Patierts \\
\hline cervical radiculopathy & 74 \\
peripheral neuropathy & 15 \\
other mononeuropathy & 13 \\
trachial ple wopathy & 3 \\
other & 30 \\
\hline
\end{tabular}

The specialty of the referring physician had a pronounced influence on the accuracy of the referring diagnosis (Table 3). A higher percentage of the patients referred by primary care physicians (family practice and internal medicine) for suspected CTS was actually found to have the disorder, more than for any of the other specialties; however, primary care referrals represented a minority of the cases seen.

\begin{tabular}{|ccc|}
\hline \multicolumn{3}{|c|}{ TABLE 3 } \\
RefERrial SOURCE FOR PATIENTS FOUND TO HAVE CTS \\
\hline \hline Referal Source & No. of Patierts & No. Riered for CT S (\%) \\
\hline $\begin{array}{c}\text { primary care physician } \\
\text { spedialty physian }\end{array}$ & 43 & $36(83.7)$ \\
\hline
\end{tabular}

The demographic characteristics of the 250 patients with CTS were not different from what would be expected. There was a slight preponderance of females (136) over males (114). The mean age of men (51.9 years, range 24-79) did not differ significantly from that of women (49.4 years, range 21-87). One hundred seventy-one patients had bilateral symptoms and 53 had unilateral symptoms. In 26 patients, only one hand was examined.

The type and number of studies necessary to make a diagnosis is currently an issue in many localities. We routinely rely on sensory nerve conduction studies, usually stimulating in the palm and recording over the median and ulnar nerves at the wrist, as the most sensitive indicators of CTS. $[5,6,8,9]$ A total of 203 patients underwent palmar sensory nerve conduction studies, and this subgroup was analyzed in detail. The data analysis presented here is derived from the most severely affected extremity, when both sides were studied.

In general, the degree of prolongation of the distal latency and the size of the nerve action potential on 
sensory nerve conduction studies correlates with the severity of the lesion. The upper limit of normal for distal latency is $2.2 \mathrm{msec}$, but a study is judged to be abnormal if the median distal latency exceeds the ipsilateral ulnar distal latency by more than $0.2 \mathrm{msec}$.[5] As shown in Fig. 1, in slightly over $25 \%$ of studies, the only abnormality was relative prolongation of the median distal latency in comparison to the ipsilateral ulnar latency, and 50\% of studies showed latency prolongations just beyond the upper limit of normal. This would indicate that the majority of cases of CTS were relatively mild. Paradoxically, we observed that the patients who showed the mildest degree of abnormality on electrophysiological testing often had the most bothersome symptoms. The symptoms in this group of patients might have been the most amenable to nonsurgical therapies.

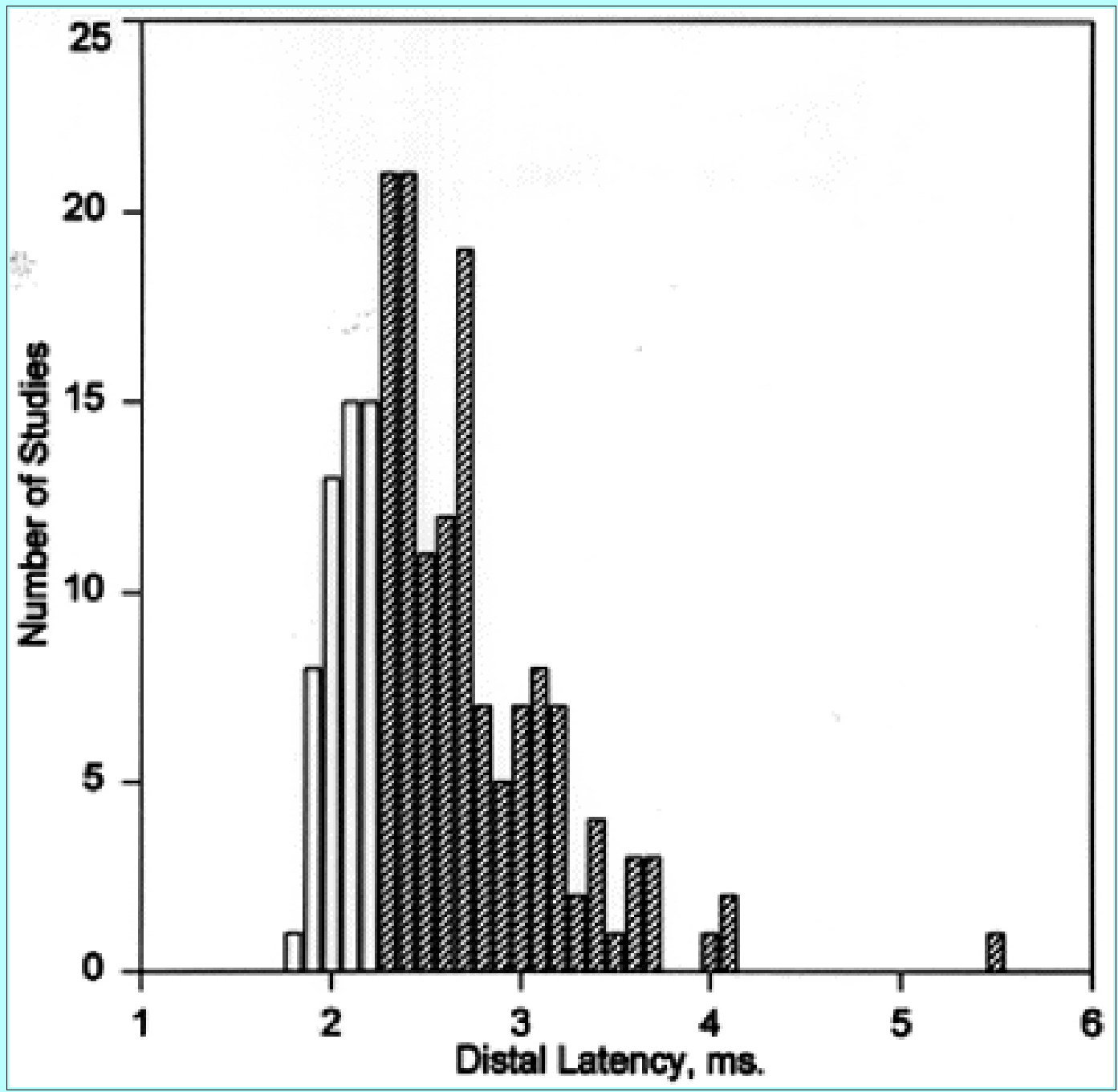

Fig. 1. Bar graph showing the distribution of median nerve distal latency values obtained by palmar sensory stimulation in the most severely affected hand of 187 patients. An additional 16 patients had no recordable median sensory response. The upper limit of normal is 2.2 msec. The unfilled bars represent those studies in which a normal absolute latency was obtained, but the median latency was significantly longer than the ipsilateral ulnar latency. This cohort represented $27.8 \%$ of the median sensory values obtained, and $50.3 \%$ of all studies had a latency of $2.4 \mathrm{msec}$ or less.

As noted earlier, a higher proportion of patients referred by primary care practitioners for CTS were found to have been correctly diagnosed than patients referred by specialists. We hypothesized that the higher "hit rate" was due to selective referral of only the most severe cases. The amplitude of the median 
palmar sensory nerve action potential in the two groups was compared. The lower limit of the normal range is $50 \mu \mathrm{V}$. In the group referred by primary care physicians, the mean value was $44.4 \mu \mathrm{V}$, and the mean of the specialist-referred cases was $67 \mu \mathrm{V}(\mathrm{p}=0.0094)$. The distributions of these results are shown in Fig. 2.

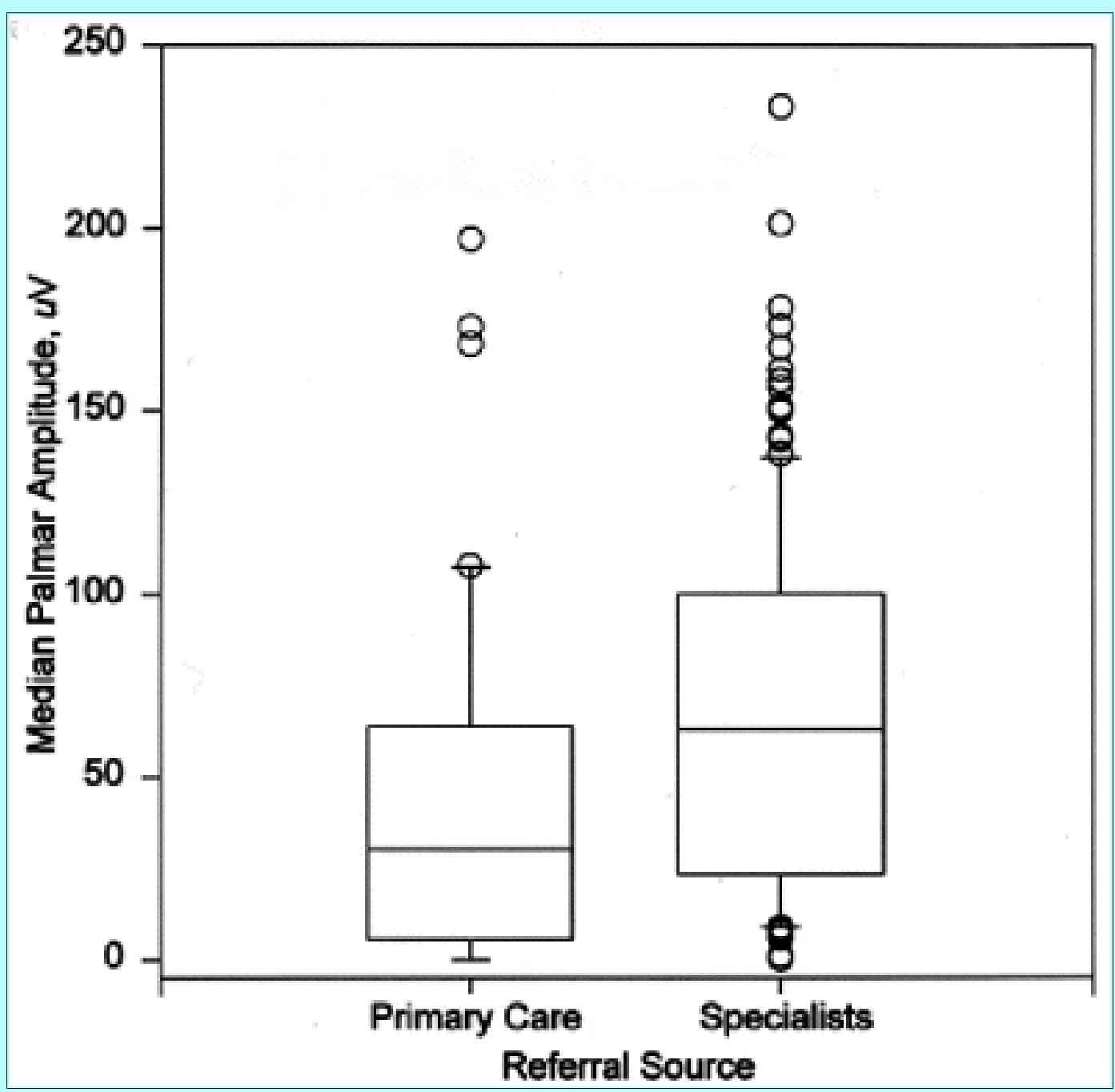

Fig. 2. Box plot graph showing the distribution of median nerve sensory amplitudes obtained with palmar stimulation in relation to the specialty of the referring physician. In this plot, the lower boundary of the box indicates the 25 th percentile, the line within the box represents the median value, and the upper boundary of the box represents the 75 th percentile of the data. The lower and upper error bars represent the 10th and 90th percentiles, respectively, and the data points beyond the error bars are outlying values. The amplitude of the median sensory nerve action potentials obtained in patients referred by primary care physicians is significantly lower than that from specialist-referred patients $(\mathrm{p}=$ 0.0094).

\section{DISCUSSION}

This study was conducted to obtain a clearer picture of the prevalence of CTS in an actual practice setting of an academic electromyography laboratory. We found that CTS was demonstrated in approximately $15 \%$ of the studies performed in a 12-month period, indicating that CTS is a significant part of routine electrodiagnostic practice. 
Analysis of these data provided some surprising results. We found that at least $50 \%$ of the patients had a relatively mild degree of abnormality. Almost $50 \%$ of the patients in whom electrodiagnostic testing confirmed CTS were originally thought to have another clinical problem by their referring physician. This suggests that mild CTS is underrecognized or that it may be mistaken for some other condition. The fact that fully one-third of patients who were thought to have CTS by the referring physician had some other diagnosis revealed by electrodiagnostic testing also attests to the fact that other disorders can masquerade as CTS. Clearly, the more accurate diagnosis provided by electrodiagnostic testing will provide the means for better targeting of treatment.

There is evidence from this study that indicates some referral bias on the part of primary care physicians. It would appear that only patients who have more significant symptoms are referred for electrodiagnostic testing. Our data do not allow us to determine which patients were referred from a primary care physician to a specialist specifically for evaluation of CTS, but we believe that the number of such patients is small. Overall, more patients were referred by specialists, and the referral diagnosis in a significant number of them was for some entity other than CTS. We would thus conclude that many patients with CTS are not recognized as having the disorder, even when they are seen by a specialist. The important difference between the specialist and the primary care physician is that the specialist is more likely to refer the patient for electrodiagnostic testing.

We believe there is a clear benefit to patients who undergo electrodiagnostic testing testing when they present with symptoms that indicate CTS. The chances for misdiagnosis and application of inappropriate treatment are significantly reduced when the objective data from appropriately performed nerve conduction and EMG studies are available. The American Association of Electrodiagnostic Medicine is presently organizing an outcome study to determine this value. Until the results of this study are available, data such as we present here will serve by inference to indicate the usefulness of electrodiagnostic testing in the patient who presents with upper-extremity pain, paresthesia, and weakness.

\section{Investment Disclosure}

The authors have no financial interest in any of the technical modalities discussed in this paper.

\section{References}

1. American Association of Electrodiagnostic Medicine, American Academy of Neurology, American Academy of Physical Medicine and Rehabilitation: Practice parameter for electrodiagnostic studies in carpal tunnel syndrome: summary statement. Muscle Nerve 16:1390-1391, 1993

2. Anonymous: Practice parameter for carpal tunnel syndrome (summary statement). Report of the Quality Standards Subcommittee of the American Academy of Neurology. Neurology 43:2406-2409, 1993

3. Anonymous: Practice parameter for electrodiagnostic studies in carpal tunnel syndrome (summary statement). American Academy of Neurology, American Association of Electrodiagnostic Medicine, and American Academy of Physical Medicine and Rehabilitation. Neurology 43:2404-2405, 1993

4. Budnick LD: Clinical strategies for work-related carpal tunnel syndrome. New Jersey Med 93:27-31, 1996 
5. Daube JR: Percutaneous palmar median nerve stimulation for carpal tunnel syndrome. Electroencephalogr Clin Neurophysiol 43:139-140, 1977 (Abstract)

6. De Lean J: Transcarpal median sensory conduction: detection of latent abnormalities in mild carpal tunnel syndrome. Can J Neurol Sci 15:388-393, 1988

7. Jablecki CK, Andary MT, So YT, et al: Literature review of the usefulness of nerve conduction studies and electromyography for the evaluation of patients with carpal tunnel syndrome. AAEM Quality Assurance Committee. Muscle Nerve 16:1392-1414, 1993

8. Jackson DA, Clifford JC: Electrodiagnosis of mild carpal tunnel syndrome. Arch Phys Med Rehabil 70:199-204, 1989

9. Kimura J: The carpal tunnel syndrome: localization of conduction abnormalities within the distal segment of the median nerve. Brain 102:619-635, 1979

10. Mainous AG III, Nelson KR: How often are preoperative electrodiagnostic studies obtained for carpal tunnel syndrome in a Medicaid population? Muscle Nerve 19:256-257, 1996 (Letter)

11. Miller RS, Iverson DC, Fried RA, et al: Carpal tunnel syndrome in primary care: a report from ASPN. Ambulatory Sentinel Practice Network. J Fam Practice 38:337-344, 1994

12. Stevens JC: AAEE minimonograph \#26: the electrodiagnosis of carpal tunnel syndrome. Muscle Nerve 10:99-113, 1987

13. Stevens JC, Sun S, Beard CM, et al: Carpal tunnel syndrome in Rochester, Minnesota, 1961 to 1980. Neurology 38:134-138, 1988

14. Tanaka S, Wild DK, Seligman PJ, et al: The US prevalence of self-reported carpal tunnel syndrome: 1988 National Health Interview Survey data. Am J Public Health 84:1846-1848, 1994

Manuscript received June 10, 1997.

Accepted in final form June 18, 1997.

Address reprint requests to: Lawrence H. Phillips, M.D., Department of Neurology, Box 394, University of Virginia, Charlottesville, Virginia 22908. email: 1hp3n@ virginia.edu. 